\title{
EchoGéo
}

22 | 2012

Varia

\section{Histoires de géographes}

Jean-Louis Chaléard

\section{(2) OpenEdition}

Journals

Édition électronique

URL : https://journals.openedition.org/echogeo/13311

DOI : 10.4000/echogeo.13311

ISSN : 1963-1197

\section{Éditeur}

Pôle de recherche pour l'organisation et la diffusion de l'information géographique (CNRS UMR 8586)

Référence électronique

Jean-Louis Chaléard, « Histoires de géographes », EchoGéo [En ligne], 22 | 2012, mis en ligne le 30 janvier 2013, consulté le 31 juillet 2021. URL : http://journals.openedition.org/echogeo/13311 ; DOI : https://doi.org/10.4000/echogeo.13311

Ce document a été généré automatiquement le 31 juillet 2021.

EchoGéo est mis à disposition selon les termes de la licence Creative Commons Attribution - Pas d'Utilisation Commerciale - Pas de Modification 4.0 International (CC BY-NC-ND) 


\title{
Histoires de géographes
}

\author{
Jean-Louis Chaléard
}

1 Un numéro « varia » n'a pas pour objectif premier de se concentrer sur un seul thème. On lira donc dans cette livraison d'ÉchoGéo des articles sur des sujets forts divers, du réchauffement climatique à la démocratie participative, des maladies infectieuses en Guyane à la «rambla » tarragonaise. Mais, au fil des rubriques, on trouve plusieurs discussions qui renvoient à des interrogations sur la géographie, sur son intérêt et ses relations avec d'autres sciences sociales.

2 La rubrique Sur le métier aborde les rapports entre histoire et géographie dont les liens anciens marquent l'enseignement de notre pays et ont influé sur l'évolution scientifique des deux disciplines. Le dossier organisé par Alexis Sierra sous forme d'entretien-débat réunit un géographe, Christian Grataloup, qui interroge l'histoire pour construire une géographie du monde, et un historien, Patrick Garcia, qui réfléchit sur la pratique de l'espace et l'usage de la géographie chez les historiens.

3 À la lumière de leur expérience et de leurs acquis sur le sujet, les intervenants nous invitent à revisiter les relations entre les deux disciplines. Partant de la situation des années 1970, ils pointent l'absence ou la faiblesse des références réciproques dans les productions scientifiques et soulignent le décalage temporel dans les approches, le rapport à la théorie et le rapport au monde. L'apport de la géographie à l'École des Annales est bien connu, mais ancien. La géographie historique a eu son heure de gloire au XIX ${ }^{e}$ siècle, avant de tomber en désuétude. Assiste-t-on à un renouvellement? Les deux interviewés insistent sur le rôle des mutations institutionnelles des années 1970 (et notamment la création des IUFM), de plusieurs revues dont EspaceTemps, qui leur ont permis de mener une réflexion sur les rapports entre l'histoire et la géographie. Celle-ci débouche sur une analyse plus large, prenant en compte la structuration, les méthodes (pensons au rôle des archives d'un côté, du terrain de l'autre) et quelques unes des évolutions récentes des deux disciplines.

4 L'entretien de Serge Weber avec les traducteurs du livre de David Harvey, Paris, capitale de la modernité, dans la rubrique Sur l'écrit, engage à poursuivre la réflexion entamée sur la géographie et ses rapports aux autres sciences sociales, à partir d'un sujet différent. David Harvey, dont la relecture de Marx a permis, depuis le début des années 1980, 
d'éclairer sous un jour nouveau le fait urbain, est à la fois géographe et philosophe. L'entretien nous laisse entrapercevoir la richesse de sa pensée qui n'est pas réductible à une lecture simpliste du marxisme. Au-delà, c'est toute une série de thèmes qui sont abordés tant sur l'évolution des courants de pensée dans le monde anglo-saxon (et le renouveau du marxisme), les problèmes posés par la traduction (comment rendre en français une pensée aussi précise et complexe, avec des concepts originaux ?) et le rôle des éditeurs indépendants (qui ouvrent une brèche dans la bibliométrie académique), engageant une réflexion plus générale sur la géographie et les sciences sociales.

En contre-point de ces dossiers, les cinq articles de cette livraison d'ÉchoGéo abordent des thèmes très divers. Pierre Pagney, fort de son exceptionnelle expérience de géographe spécialiste du climat, reste dans la veine réflexive sur la discipline, en s'interrogeant sur la climatologie française et la place des géographes dans le débat sur l'étude du réchauffement climatique. Le texte de Valérie Morel, qui porte sur la "vulnérabilité du territoire littoral guyanais aux maladies infectieuses à transmission vectorielle ", pose des questions de méthode et conclut sur l'intérêt de recherches pluridisciplinaires qui ouvrent de nouvelles possibilités d'action pour la gestion des risques sanitaires. Les trois autres textes sont à la charnière de questions sociales et urbaines. Le texte de Philippe Gervais-Lambony sur les «conditions spatiales de la démocratie participative. "Meetings » à Vosloorus » est peut-être celui qui renvoie le plus aux thèmes développés dans le dossier sur David Harvey, par son analyse des relations entre démocratie locale, société et espace. Hayuning Anggrahita aborde les inégalités socio-spatiales à partir d'une entrée sur l'approvisionnement en eau à Djakarta. L'article de Jean Rieucau sur «la promenade publique géosymbole de l'urbanité espagnole » montre, à partir de l'exemple de la Rambla Nova de Tarragone, l'originalité de cette forme urbaine qui se « corporise » et se « mondialise».

6 Si la question des relations de la géographie avec d'autres disciplines est au cœur de ce numéro d'ÉchoGéo, l'urbain y occupe également une grande place, témoignant de la vitalité des études dans ce champ scientifique. La ville apparaît dans la rubrique Sur le métier, à propos des recherches où se rencontrent géographes et historiens, dans la rubrique Sur l'écrit bien sûr avec les travaux de David Harvey et dans la majorité des articles du varia. La rubrique Sur l'image n'échappe pas à la tendance, avec un dossier sur Moscou "à l'ère de la compétition métropolitaine ». À partir de photographies récentes, les auteurs (Élisabeth Bonnet-Pineau et Aurélien Delpirou) nous présentent les métamorphoses d'une ville mondialisée mais qui reste, et doit rester pour le pouvoir politique, profondément russe.

$7 \quad$ Le dernier texte paru dans la rubrique Sur le vif de Stéphane Dovert et Frédéric Durand sur « les élections à Timor-est, 2001-2012» conforte les propos que nous avons tenus sur l'importance et l'intérêt de la géographie électorale dans le numéro 20 d'ÉchoGéo. L'article dégage la signification de ces élections qui devraient permettre le retrait prochain des troupes de l'ONU du territoire, marqué par une longue lutte pour son indépendance contre l'Indonésie. Les auteurs soulignent également quelques singularités timoraises à partir des résultats des élections et des comportements des électeurs. Leur analyse à la fois géographique et historique montre l'intérêt d'un recours conjoint à ces disciplines pour la compréhension du monde contemporain, illustrant les débats du dossier Sur le métier. Car la question des relations entre histoire et géographie est loin d'être close. 